\begin{tabular}{|l|l|l|}
\hline \multicolumn{2}{|c|}{ PublisherInfo } \\
\hline \hline PublisherName & $:$ & BioMed Central \\
\hline \hline PublisherLocation & $:$ & London \\
\hline \hline PublisherImprintName & $:$ & BioMed Central \\
\hline \hline
\end{tabular}

\title{
Profiling the flavonoid pathway
}

\begin{tabular}{|l|l|l||}
\hline \multicolumn{2}{|c|}{ ArticleInfo } \\
\hline \hline ArticleID & $:$ & 3568 \\
\hline \hline ArticleDOI & $:$ & $10.1186 /$ gb-2000-1-1-reports034 \\
\hline \hline ArticleCitationID & $:$ & reports034 \\
\hline \hline ArticleSequenceNumber & $:$ & 59 \\
\hline \hline ArticleCategory & $:$ & Paper report \\
\hline \hline ArticleFirstPage & $:$ & 1 \\
\hline \hline ArticleLastPage & $:$ & 4 \\
\hline \hline & & RegistrationDate : 2000-3-7 \\
\hline ArticleHistory & $:$ & Received \\
& $: 2000-3-7$ \\
\hline \hline ArticleCopyright & $:$ & BioMed Central Ltd2000 \\
\hline \hline ArticleGrants & $:$ & \\
\hline \hline \hline ArticleContext & $:$ & 130591111 \\
\hline \hline
\end{tabular}




\section{Abstract}

GeneCalling, a new expression profiling technique, has been used to elucidate regulatory gene networks in plants.

\section{Significance and context}

Flavonoids are plant secondary metabolites with roles in processes such as pigmentation, defense responses and protection from ultraviolet-B radiation. In maize, biosynthesis of the flavonoid anthocyanin is known to be controlled by members of the $M Y B$-domain family of transcriptional regulators, $C 1$ and $P$, and the basic helix-loop-helix domain proteins $\mathrm{R}$ and $\mathrm{B}$. $\mathrm{C} 1$ acts with $\mathrm{R}$ to induce the expression of downstream targets such as Chalcone synthase $(C 2)$ and Flavone/dihydroflavonol $N A D P H$-dependent reductase $(A 1)$, whereas $P$ can act individually to activate targets such as $A 1$. To elucidate further the pathway of anthocyanin biosynthesis, Bruce et al. have used CuraGen's GeneCalling technology, an mRNA profiling technique, to identify genes that are upregulated or downregulated on expression of a $C 1 / R$ fusion construct (CRC) or $P$. This technique, unlike DNA microarray technology, does not require prior knowledge of the target gene sequences. The authors transformed suspension cells from the maize inbred line Black Mexican Sweet (BMS) with estrogeninducible expression constructs of $C 1 / R$ and $P$, and assayed changes in gene expression in the maize cells at 0,6 and 24 hours after estrogen treatment using the GeneCalling methodology. Only genes that showed at least a twofold change in expression were analyzed further.

\section{Key results}

In the CRC lines, 663 cDNAs were differentially expressed after estrogen treatment, compared with 217 in the lines transformed for the $P$ locus. Comparison of gene expression profiles from all lines identified six genes that showed altered expression in response to expression of both $\mathrm{C} 1 / \mathrm{R}$ and $\mathrm{P}$. These included $A 1$, a known target of $\mathrm{C} 1 / \mathrm{R}$, and Phenylalanine ammonia-lyasel (PAL1), which was known to be involved in flavonoid biosynthesis, but had not been shown previously to be regulated by $\mathrm{C} 1 / \mathrm{R}$ or $\mathrm{P}$. The common targets also included an enolase and a glutathione $\mathrm{S}$-transferase that was $58 \%$ identical to Bronze2 $(\mathrm{Bz} 2)$, a downstream target of $\mathrm{C} 1 / \mathrm{R}$ that acts in the anthocyanin pathway. The accuracy of GeneCalling in estimating the degree of gene induction or repression was tested by analysis of mRNA expression in the same circumstances by northern blotting. For some genes identified in this study (for 
example, C2, Flavanone 3-hydroxylase, A2, encoding proanthocyanidin synthase, and Bronze1, which encodes UDP-glucose:flavonoid 3-O glucosyltransferase), hybridization with gene-specific probes yielded results similar to those of GeneCalling. Northern blots did not, however, detect a change in expression of glutathione $S$-transferase and the enolase, which GeneCalling had shown to be differentially expressed. Bruce et al. suggest that GeneCalling is more allele-specific than northern blotting, a technique more likely to mask expression changes by cross-reacting with closely related genes.

\section{Methodological innovations}

GeneCalling comprises techniques that analyze and group the outputs from expression studies so that they can be readily compared and changes in gene expression identified. The cDNAs generated from each of the sample mRNAs are each digested with up to 72 pairs of restriction enzymes. The digested DNA is ligated to adaptors and then amplified with fluorescently tagged primers. Amplified products are electrophoresed and the fragment size profile for each sample is recorded. GeneCalling computational tools then attempt to match the restriction site polymorphisms to sequences in a database. The pattern of restriction site polymorphisms defined by the enzyme digests acts as a molecular fingerprint of each cDNA. The specific algorithms and search parameters are not described, but for this study it is claimed that around 6,000-8,000 unique cDNAs were represented in the sample of over 19,000 fragments (ranging from 50-500 base-pairs in length) that were analyzed, suggesting that the number of restriction digests was sufficient to ensure complete coverage of the sample mRNA population.

\section{Links}

Some additional information on GeneCalling can be found at the CuraGen Corporation website.

\section{Conclusions}

The results obtained by Bruce et al. with GeneCalling confirm some of the regulatory interactions for genes regulated by $C 1 / R$ and $P$ that have been reported previously, providing independent confirmation of these results as well as validation of the GeneCalling technology. Potential new downstream targets were found and a temporal profile of gene expression after $C 1 / R$ or $P$ induction was created that identifies, for example, early- versus late-acting genes.

\section{Reporter's comments}


In theory, GeneCalling provides a fast and sensitive assay for differential gene expression that does not require prior knowledge of gene sequences being assayed. The hardware requirements are likely to be found in most molecular biology labs (PCR machine, sequencing apparatus, computer), although the exact specifications of the equipment used by Bruce et al. were not described. The cost of GeneCalling presumably lies in access to the proprietary technology and computational tools. A limitation of the technique arises when fragment matches cannot be made to a database of known sequences. In this case, the fragment must be isolated and sequenced and the corresponding cDNA cloned to obtain full-length sequence, as for conventional differential display studies. So, for poorly characterized genomes, the advantage of this technology lies in the sensitivity of the assay for differential expression rather than in gene identification per se.

\section{Table of links}

Plant Cell

CuraGen Corporation

\section{References}

1. Bruce W, Folkherts O, Garnaat C, Crasta O, Roth B, Bowen B: Expression profiling of the maize flavonoid pathway genes controlled by estradiol-inducible transcription factors CRC and P. Plant Cell. 2000, 12: 65-79. 1040-4651 